\title{
Metabolic Syndrome in the Indian Population: Public Health Implications
}

\author{
PP Mohanan
}

\begin{abstract}
Metabolic syndrome (MS) is now being increasing recognized as an emerging threat which will invade desktops of public health policy planners in the decades to come. The clusters which make this syndrome and its etiopathogenesis will keep getting varied in different ethnic populations, regions and countries. Factors like migration, socioeconomic status, lifestyle, nutrition habits play important role. Therefore, research in MS provides an interdisciplinary forum to explore the pathophysiology, recognition, and treatment of the cluster of conditions associated with the evolving entity of MS.
\end{abstract}

Keywords: Atherosclerotic, Dyslipidemia, Intramyocellular lipids.

How to cite this article: Mohanan PP. Metabolic Syndrome in the Indian Population: Public Health Implications. Hypertens J 2016;2(1):1-6.

\section{Source of support: Nil}

\section{Conflict of interest: None}

\section{INTRODUCTION}

A global transition in the disease pattern has been observed, where the relative impact of infectious diseases is decreasing while chronic diseases such as cardiovascular disease (CVD) and diabetes are increasingly dominating the disease pattern. ${ }^{1}$ Epidemiologists in India and international agencies such as the World Health Organization (WHO) have been sounding an alarm on the rapidly rising burden of CVD for the past 15 years. It is estimated that by 2020, CVD will be the largest cause of disability and death in India, with 2.6 million Indians predicted to die due to CVD. ${ }^{2,3}$ Metabolic syndrome (MS) is now being increasingly recognized as an emerging threat that will invade desktops of public health policy planners in the decades to come. The clusters that make this syndrome and its etiopathogenesis will keep getting varied in different ethnic populations, regions, and countries. Factors such as migration, socioeconomic status, lifestyle, and nutrition habits play an important role.

\section{Director and Head \\ Department of Cardiology, Westfort Hi-Tech Hospital, Thrissur Kerala, India}

Corresponding Author: PP Mohanan, Director and Head Department of Cardiology, Westfort Hi-Tech Hospital, Thrissur Kerala, India, Phone: +919846076006, e-mail: drppmohanan@ gmail.com
Therefore, research in MS provides an interdisciplinary forum to explore the pathophysiology, recognition, and treatment of the cluster of conditions associated with the evolving entity of MS. These include but are not limited to central obesity, endothelial dysfunction, insulin resistance (IR), dyslipidemia, glucose intolerance, type 2 diabetes, prothrombotic and pro-inflammatory states, hyperinsulinemia, hyperuricemia, hypertension, CVD, and polycystic ovarian syndrome (PCOS). Interaction of various factors, which could contribute to IR, diabetes, and coronary heart disease (CHD), is shown in Figure 1.

\section{DEFINING MS}

Metabolic syndrome represents as a constellation of interconnected physiological, biochemical, clinical, and metabolic risk factors, including hypertension, dyslipidemia, central obesity, glucose intolerance, proinflammatory, and pro-thrombotic states, which reflect an underlying IR. Various bodies such as the $\mathrm{WHO}^{4}$ the National Cholesterol Education Program-adult treatment panel III (NCEP-ATP III $)^{5}$ and the International Diabetes Federation (IDF) ${ }^{6}$ share common ground to define MS by acknowledging the disorders of glucose metabolism, hypertension, dyslipidemia, and obesity. These underlying mechanisms are exacerbated by the complex interplay between age, genetic conditioning, and an inappropriate lifestyle comprising sedentary

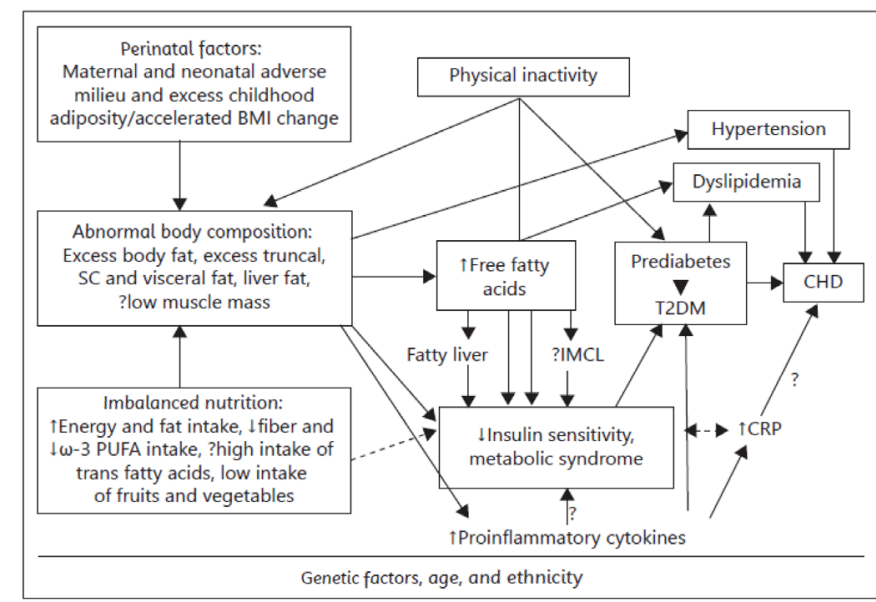

Fig. 1: Complex interactions of genetic, perinatal, nutritional and other acquired factors in development of IR, T2DM and CHD in Asian Indians. CRP: C-reactive protein; IMCL: Intramyocellular lipids; SC: Subcutaneous 
lifestyle and surplus availability of energy dense saltenriched food.

The emerging typical Asian Indian urban/migrant has phenotype of higher percentage of body fat at a lower value of body mass index (BMI), high waist-hip ratio (WHR) at a relatively low waist circumference, and less lean body mass as compared with ethnic groups. Asian Indian migrants have higher values of BMI and WHR and thicker skinfolds than urban subjects in India; Asian Indian men had significantly thicker truncal skinfolds than Caucasians. High body fat, often at BMI values that are in nonobese range, is another characteristic phenotypic feature of Asian Indians. This leads to abnormal lean body mass (muscle) to fat ratio and sarcopenia with higher body fat composition. The emerging Asian Indian phenotype is high body fat with relatively less body BMI, less lean body mass (particularly in lower limbs), high BF/BMI ratio (higher body fat per unit BMI), high WHR (absolute value of waist circumference may not be excessive), variable subscapular/triceps ratio, and high intramyocellular lipids. ${ }^{\text {? }}$

\section{Diagnostic Criteria for MS in Indians}

The diagnostic criteria for MS in Indians are listed as follows (Table 1):

- Waist circumference in Indian men: greater than 35"; Indian women: greater than 31"

- High triglyceride: Equal to or greater than $150 \mathrm{mg} / \mathrm{dl}$

- Low HDL cholesterol: $<40 \mathrm{mg} / \mathrm{dl}$ for men, $<50 \mathrm{mg}$ / dl for women

- High blood pressure: equal to or greater than $130 / 85$ $\mathrm{mm} \mathrm{Hg}$

- High fasting blood glucose: $100 \mathrm{mg} / \mathrm{dl}$ (pre-diabetes)

\section{Epidemiology of MS}

Metabolic syndrome is a complex web of metabolic factors that are associated with a two-fold risk of CVD and a five-fold risk of diabetes. Individuals with MS have a
30 to $40 \%$ probability of developing diabetes and/or CVD within 20 years, depending on the number of components present. In the United States (US), the prevalence of the MS in the adult population was estimated to be more than $25 \%$. Similarly, the prevalence of MS in European countries was approximately $23 \%$. It was estimated that 20 to $25 \%$ of South Asians have developed MS and many more may be prone to it. ${ }^{8}$ The main reason why MS is attracting scientific and commercial interest is that the factors defining the syndrome are all factors associated with increased morbidity and mortality in general and from CVD in particular.

Rapid demographic, nutritional, and economic changes are occurring in South Asians. The life expectancy and the percentage of elderly population have increased. Most importantly, globalization of diets and consumption of nontraditional fast food have occurred at a rapid pace in urban areas. Furthermore, these dietary changes are most noticeable in children. In South Asian countries, a rapid increase in western fast food outlets, sale of aerated sweet drinks, and increased consumption of fried snacks in school children is being commonly seen. In addition, South Asians are less physically active, and sedentary lifestyle is increasing, particularly in children. Further, migration from villages to cities is increasing. These intracountry migrants become urbanized, mechanized, resulting in nutritional imbalance, physical inactivity, stress, and increased consumption of alcohol and tobacco.

Nationally representative studies regarding the prevalence of the MS are generally not available from any South Asian country. Available data indicate that the prevalence of the MS in Asian Indians varies according to region, extent of urbanization, lifestyle patterns, and socioeconomic/cultural factors. Recent data show that about one-third of the urban population in India's major cities have MS. ${ }^{9}$ The National Cholesterol Education Programme (NCEP) definition is more flexible, as it can diagnose MS even in the absence of glucose

Table 1: Clinical diagnostic criteria for MS

\begin{tabular}{llll}
\hline Risk factor & WHO & Updated NCEP & Asian Indians/IDF \\
\hline Fasting plasma glucose & & $\geq 100 \mathrm{mg} / \mathrm{dl}$ & $\geq 100 \mathrm{mg} / \mathrm{dl}$ \\
Abdominal obesity & $>102 / 94 \mathrm{~cm}-$ Men & $>102 \mathrm{~cm}-\mathrm{Men}$ & $>90 \mathrm{~cm}-$ Men \\
- Waist circumference & $>88 / 80 \mathrm{~cm}-$ Women & $>88 \mathrm{~cm}-$ Women & $>80 \mathrm{~cm}-$ Women \\
- Body mass index & $>25 \mathrm{~kg} / \mathrm{m}^{2}$ & - & $>23 \mathrm{~kg} / \mathrm{m}^{2}$ \\
- Waist hip ratio* & $>0.90-$ Men & & $>0.9-$ Men \\
& $>0.85-$ Women & & $>0.8-$ Women \\
Dyslipidemia & $<35 \mathrm{mg} / \mathrm{dl}-$ Men & $<40 \mathrm{mg} / \mathrm{dl}-$ Men & $<40 \mathrm{mg} / \mathrm{dl}-\mathrm{Men}$ \\
- Triglyceride & $<30 \mathrm{mg} / \mathrm{dl}-$ Women & $<50 \mathrm{mg} / \mathrm{dl}-$ Women & $<50 \mathrm{mg} / \mathrm{dl}-$ Women \\
- High-density lipoprotein & $>150 \mathrm{mg} / \mathrm{dl}$ & $\geq 150 \mathrm{mg} / \mathrm{dl}$ & $\geq 150 \mathrm{mg} / \mathrm{dl}$ \\
- Cholesterol & $\geq 140 / \geq 90 \mathrm{~mm} \mathrm{Hg}$ & $\geq 130 / \geq 85 \mathrm{~mm} \mathrm{Hg}$ & $>130 />85 \mathrm{~mm} \mathrm{Hg}$ \\
- Blood pressure & & \\
*Misra et al JAPI 2009 & &
\end{tabular}


intolerance, which in itself is a predisposition to dysmetabolic dyslipidemia, an obesity phenotype, and pro-inflammatory status.

Chow et $\mathrm{al}^{10}$ found a prevalence of MS of $26.9 \%$ in males and $18.4 \%$ in females in southern India. The prevalence of MS did not change with respect to age difference; 20 to 40 and 41 to 60 age groups showed similar prevalence of MS, and a marginal decrease was seen in more than 60 age group. The prevalence of MS based on ATP III criteria in Jaipur (urban north Indian population) was $24.9 \%$. In Chennai urban population study (CUPS), ${ }^{11}$ the prevalence of MS as defined by European group for the study of insulin resitance (EGIR) was found to be $11.2 \%$. In the study of 10 industrial settings, prevalence of MS was $26.6 \%$. Among Asian Indian, immigrants living in US showed the prevalence of MS to be $32 \%$. These findings suggest that there is a significant difference even within an urban environment in different socioeconomic groups. There were no significant differences between genders in all age groups, except for individuals aged more than 70 years, in which there was a predominance of females (65.4\%). Men and women aged 40 to 59 years were three times more likely to have MS than those ranging in age from 20 to 39 years. In men aged more than 60 years, this probability was four times higher, and in women of the same age, the chance was increased by six times.

\section{Consequences of the MS}

The phenotype of obesity and body fat distribution are distinctive in South Asians and are important contributory factors for development of IR and the MS. The development of obesity, or more specifically an increase in abdominal fat, is thought to be the primary event in the progression of MS. A tendency to gain fat in the abdominal area, as opposed to the hip, buttock, and limb areas, is linked to a rise in fatty acids in the blood, which is thought to lead to IR, high blood pressure, abdominal blood lipids, and eventually diabetes. It is also more susceptible to lipolysis than subcutaneous fat and is associated with systemic inflammatory response. The homeostatic model assessment (HOMA) index is an alternative for the assessment of IR, mainly because it is a fast, easily applicable in epidemiological studies, and low-cost method. The HOMA index has proved to be a robust clinical and epidemiological tool in descriptions of the pathophysiology of diabetes. Homeostatic model assessment analysis allows assessment of inherent beta cell function and insulin sensitivity and can characterize the pathophysiology in those with abnormal glucose tolerance. Enas et $\mathrm{al}^{12}$ in coronary artery disease in Indians (CADI) study report the prevalence of diabetes to be three to six times higher among South Asians than Europeans, Americans, and other Asians. In India, it is estimated that 32 million people suffer from diabetes, and the number is projected to increase to 69.8 million by 2025 .

Hypertension is also a central component of MS, with approximately $85 \%$ of MS patients suffering from this condition. Hypertension is usually diagnosed at a late stage in the disease, at which point consequential life- threatening diseases, such as kidney damage and heart failure, occur. Insulin resistance and obesity have been recognized as the leading cause of hypertension. Fifty per cent of hypertensive people are insulin resistant. Obesity and IR contribute to the development of hypertension, both independently and collectively. Under normal, healthy circumstances, introduction of insulin into the bloodstream causes a release of nitric oxide and subsequent vasodilation. This is not seen in obese, insulin-resistant individuals. Instead, IR and thus compensatory hyperinsulinemia activate the reninangiotensin-aldosterone system with consequential vasoconstriction and hypertension.

Increased prevalence of low high density lipoprotein cholesterol (HDL-C) has been reported earlier by Enas et al. ${ }^{12}$ World Health Organization found that only $4 \%$ of Asian Indian men and 5\% Asian Indian women had optimal HDL-C levels. Low HDL-C levels are a strong predictor of occurrence and reoccurrence of myocardial infarction (MI) and stroke and are associated with risk of premature and severe coronary artery disease (CAD). At the forefront is apolipoprotein (Apo) A Apo B Apo A ratio Apo $\mathrm{B} / \mathrm{Apo} \mathrm{A} 1$ ratio. Apolipoprotein $\mathrm{B}$ is the major apolipoprotein found in low-density lipoprotein (LDL), intermediate-density lipoprotein (IDL), and very LDL (VLDL), and it is the primary ligand for the LDL receptor. Apolipoprotein A1 is the major protein constituent of HDL. The Apo B/Apo A1 ratio provides an atherogenic to antiatherogenic lipoprotein ratio that has been shown to be a better predictor of CVD than LDL level and HDL level. The Apo B/Apo A1 ratio can identify individuals with preponderance of small dense LDL particles.

Lipoprotein a $[\operatorname{Lp}(\mathrm{a})]$, considered as an emerging risk factor by NCEP ATP III, has been implicated in the development of the premature atherosclerotic disease seen in South Asians. Among patients with Lp(a) excess, the CAD risk is increased by three-fold in the absence of other risk factors. The risk increases to eight-fold with low HDL-C, 12-fold with high LDL-C, 16-fold with diabetes and 25-fold with TC/HDL-C ratio. The higher the Lp(a) level, the lower the age of first heart attack, and the most affected individuals develop MI by the third to fifth decade of life. High levels of Lp(a) correlate with the prematurity, severity, extent, and progression of coronary 
atherosclerosis as well as the occurrence and recurrence of MI among Asian Indians. ${ }^{13}$

The CV field has recently shown great interest in the role of inflammation in the development of ASCVD. The basic concept is that atherogenesis represents a state of chronic inflammation. The findings that elevations of serum c reactive protein (CRP) carry predictive power for the development of major CV events led to the concept that advanced and unstable atherosclerotic plaques are in an even higher state of inflammation than stable plaques. In our study, females (10.2\%) had higher rates of elevated CRP than males (6.43\%). It is of interest that obese persons and particularly those with MS also have elevated levels of CRP. ${ }^{14}$ It was found that of the total subjects with elevated CRP, $86.95 \%$ were found to be obese.

Although it is well known that conventional risk factors (many of which are included as components of MS) can predict type 2 diabetes and CHD on their own, there are evidences to suggest that the MS may add to the prediction of type 2 diabetes and CHD beyond that provided by individual components. Subjects with the MS from western countries face a two-fold greater risk of all-cause mortality and a two- to three-fold increased risk of CV mortality than those without the syndrome. ${ }^{15}$ Metabolic syndrome also predicts diabetes independently of other factors. Although the clinical validity of MS to predict type 2 diabetes and, in particular, CHD has been debated by several authors, studies show that odds ratios (ORs) for predicting incident CHD using different definitions of the MS [NCEP ATP III (OR 2.00), IDF (1.69), and the WHO (OR 1.73)] were almost similar, independent of age, sex, ethnic origin, history of CHD and type 2 diabetes mellitus (T2DM), non-HDL-C, smoking status, and family history of MI. Subjects free from CHD and/or CHD risk equivalents, when evaluated with NCEP ATP III, IDF, and WHO definitions, were also shown to have similar ORs for predicting CHD.

The Framingham risk score (FRS) has traditionally been used as a predictor of the 10-year risk of CHD and takes into account age, smoking status, lipid profile, blood pressure, and presence or absence of DM. It predicts the 10-year CV risk of having cardiac events and categorizes patients into low (risk $<10 \%$ ), intermediate (risk 10-20\%), and high-risk groups ( $>20 \%)$. The incremental value of MS over the traditionally used FRS as a marker of future $\mathrm{CV}$ events is debatable, with studies suggesting that it may be inferior to FRS in predicting this risk. However, as FRS is dependent on age and estimates only the 10-year $\mathrm{CV}$ risk, it may underestimate the overall risk in young patients (with a longer life expectancy) as well as in those with MS, many of whose individual components are not included in the risk score. ${ }^{16}$

\section{Prevention and Control of MS in India}

The high levels of risk observed justify the need for establishing a surveillance system to monitor the trends of MS and ASCVD risk factors over time. Continuing surveillance efforts would provide us with an opportunity to develop evidence-based, cost-effective CVD prevention, detection, and management strategies. Early intervention, particularly with lifestyle change, would delay the onset of advance forms of MS and a high-risk status.

- Intensive efforts should be made to make South Asians aware that they are at a high risk for development of T2DM and CHD. The health education programs should be directed toward all socioeconomic strata of society.

- The preventive measures should be particularly vigorous for those with the family history of T2DM and/or premature CHD.

- The therapeutic lifestyle changes should be encouraged from the childhood. The school curriculum should be suitably modified to include messages related to healthy nutrition and physical exercise. Regular physical activity should be strongly advised and television and internet usage should be restricted.

- Body weight and anthropometric indices should be maintained within normal limits as given below. The physicians should be made aware that these provisional limits for defining normal BMI and waist circumference might be revised in the future.

- On the basis of the recent data, and provisional recommendations of $\mathrm{WHO}, \mathrm{BMI}$ should be maintained between 19 and $23 \mathrm{~kg} / \mathrm{m}^{2}$.

- The waist circumference should be maintained below $90 \mathrm{~cm}$ for men and $80 \mathrm{~cm}$ for women.

- Overweight individuals and those with abdominal obesity should be actively managed to lose weight by lifestyle measures.

- Detection of one component of the MS should lead to search for the other components and appropriate management.

- Currently, no drug (e.g., metformin) is recommended for management of IR and the MS. However, these guidelines may change in future, particularly for those who have impaired glucose tolerance (IGT), in particularly in view of recent evidence from a prevention trial from India.

- Adequate nutrition during the intrauterine period should be given to prevent early-life adverse events, which may promote IR in adulthood. 


\section{Physical Activity Guidelines for $\mathbf{M S}^{17}$}

- Physical inactivity should be avoided as far as possible.

- Pre-participation medical consultation is recommended for those with chronic conditions or those who are symptomatic. Inactive people should start slow and gradually increase physical activity.

- Brisk walking (walking at an intensity wherein an individual finds speaking difficult but not impossible) is the preferred initial mode of exercise, as this does not require any special training or equipment.

- In general, a total of 60 minutes of physical activity is recommended every day; this includes aerobic activity, work-related activity, and muscle-strengthening activity.

- Physical activity can be accumulated throughout the day in blocks as short as 10 minutes. Work-related activity should be encouraged wherever possible.

- There is a dose-response relationship between physical activity and health; greater benefit is derived by exceeding minimum recommendations. For additional and more extensive health benefits, adults can increase their aerobic physical activity to 300 minutes (5 hours) a week of moderate intensity, or 150 minutes a week of vigorous-intensity aerobic physical activity. This issue should also be researched in Asian Indians.

- Physical activity must be individualized on the basis of person's abilities and comorbidities. Much like pharmacological therapy, it requires prescription with careful consideration of both appropriate dosage and frequency. Dynamic yoga should be encouraged but needs more research.

- Children should undertake at least 60 minutes of outdoor physical activity. Screen time (television/ computers) should be less than 2 hours a day.

Dietary modifications, including a reduction in intake of saturated fat and refined carbohydrates and sweetened beverages, help in modifying childhood obesity. In countries in nutritional transition, including India, it is seen that adolescents tend to favor high carbohydrate and saturated fat intake. Dietary strategies should highlight the need to stick to traditional diets and restriction of sweetened beverages, high saturated, and transfat, containing food stuffs. Snacking between major meals should be discouraged. Low-fat dairy food should be encouraged. Avoidance of salted and processed food is essential to prevent hypertension. ${ }^{18}$ Increased dietary fiber and including green leafy vegetables and fruits need to be encouraged to help prevent obesity. It is now also appreciated that nutrition during perinatal period and early childhood may influence IR and MS in later life.
Hence, both maternal undernutrition and excess nutrition in children have deleterious effects and need to be avoided.

As one of the important components of diabetes and CVD is IR, medications that improve the IR have been shown to have a beneficial effect. Trials with metformin, acarbose, and glitazones have shown some promising results. Newer agents, such as glucagon-like peptide (GLP)-1, dipeptidyl peptidase (DPP)-IV inhibitors, and the endocannabinoid receptor blocker rimonobant, have also demonstrated promising but variable results. At present, we do not have a single common mechanism for the syndrome and there is no single effective treatment. Effective management of obesity, dyslipidemia, hypertension, and dysglycemia has shown benefit. Dyslipidemia plays a major role in the pathophysiology of the MS, and its correction is a primary goal in order to prevent CVD in patients with the MS. Statins and fibrates are the most effective drugs for controlling dyslipidemia associated with IR. Statins act by reducing LDL-C concentrations, while fibrates decrease levels of triglycerides and small, dense LDL particles and increase HDL-C concentrations. Ezetimibe, a drug of the azetidinone family that selectively inhibits intestinal cholesterol absorption, is effective in potentiating the hypocholesterolemic effects of statins. In combination with fibrates, it is a promising alternative for achieving overall control of the MS hyperlipidemia with significant reductions in LDL-C and non-HDL-C.

\section{CONCLUSION}

Metabolic syndrome has been highlighted as a major socioeconomic problem throughout India, as the burden of MS along with its individual risk factors, that is central obesity, IR, dyslipidemia, and hypertension, is evident throughout urban and rural areas studied. The additive effect of each MS component contributing to full-blown MS and also the risk of CVD and T2DM increase with increasing MS components. It is possible, therefore, that a decrease in one or two of the components of MS could potentially reduce the risk of CVD and T2DM. This would not only help save lives but would also reduce the very substantial burden that each of these conditions places on the health care systems throughout the world especially India. Accurate and timely diagnosis and treatment of MS - or better still, prevention is therefore not only crucial to the health of the world's population but also to the global economy.

\section{REFERENCES}

1. Borch-Johnsen K. The metabolic syndrome in a global perspective: the public health impact. Dan Med Bull 2007 May;54(2):157-159. 
2. Goenka S, Prabhakaran D, Ajay VS, Reddy KS. Preventing cardiovascular disease in India-translating evidence to action. Curr Sci 2009;97(3):367-377.

3. Reddy KS, Prabhakaran D, Chaturvedi V, et al. Methods for establishing a surveillance system for cardiovascular diseases in Indian industrial populations. Bull World Health Organ 2006 Jun;84(6):461-469.

4. Alberti KG, Zimmet PZ. Definition, diagnosis and clasification of diabetes mellitus and its complications-Part 1: diagnosis and classification of diabetes mellitus provisional report of a WHO consultation. Diabet Med 1998 Jul;15(7):539-553.

5. Grundy SM, Cleeman JI, Daniels SR, Donato KA, Eckel RH, Franklin BA, Gordon DJ, Krauss RM, Savage PJ, Smith SC Jr, et al. Diagnosis and management of the metabolic syndrome: An American Heart Association/National Heart, Lung, and Blood Institute Scientific Statement. Circulation 2005 Oct 25;112(17):2735-2752.

6. Alberti KG, Zimmet P, Shaw J. The metabolic syndromea new worldwide definition. Lancet 2005 Sep 24-30;366(9491): 1059-1062.

7. Joshi SR. Identification and diagnostic criteria of insulin resistance and metabolic syndrome. In: Joshi SR, editor. Primer of insulin resistance. 1st ed. Mumbai: Asian Health Care; 2003. p. 29-32.

8. Eapen D, Kalra GL, Merchant N, Arora A, Khan BV. Metabolic syndrome and cardiovascular disease in South Asians. Vasc Health Risk Manag 2009;5:731-743.

9. Misra A, Khurana L. The metabolic syndrome in South Asians: epidemiology, clinical correlates and possible solutions. Int Diabetes Monit 2009;21(3):92-101.

10. Chow CK, Naidu S, Raju K, Raju R, Joshi R, Sullivan D, Celermajer DS, Neal BC. Significant lipid, adiposity and metabolic abnormalities amongst 4535 Indians from a developing region of rural Andhra Pradesh. Atherosclerosis 2008 Feb;196(2):943-952.
11. Mohan V, Shanthirani S, Deepa R, Premalatha G, Sastry NG, Saroja R. Intra-urban differences in the prevalence of the metabolic syndrome in southern India-the Chennai Urban Population Study (CUPS No. 4). Diabetic Med 2001 Apr;18(4):280-287.

12. Enas EA, Yusuf S, Mehta JL. Prevalence of coronary artery disease in Asian Indians. Am J Cardiol 1992 Oct 1;70(9): 945-949.

13. Enas EA, Chacko V, Pazhoor SG, Chennikkara H, Devarapalli HP. Dyslipidemia in South Asian patients. Curr Atheroscler Rep 2007 Nov;9(5):367-374.

14. Grundy SM. Obesity, metabolic syndrome, and cardiovascular disease. J Clin Endocrinol Metab 2004 Jun;89(6):25952600.

15. Lakka HM, Laaksonen DE, Lakka TA, Niskanen LK, Kumpusalo E, Tuomilehto J, et al. The metabolic syndrome and total and cardiovascular disease mortality in middleaged men. JAMA 2002 Dec 4;288(21):2709-2716.

16. Wannamethee SG, Shaper AG, Lennon L, Morris RW. Metabolic syndrome vs Framingham Risk Score for prediction of coronary heart disease, stroke, and type 2 diabetes mellitus. Arch Intern Med 2005 Dec 12-26;165(22): 2644-2650.

17. Misra A, Chowbey P, Makkar BM, Vikram NK, Wasir JS, Chadha D, Joshi SR, Sadikot S, Gupta R, Gulati S, Munjal YP. For Consensus Group: Consensus Statement for Diagnosis of Obesity, Abdominal Obesity and the Metabolic Syndrome for Asian Indians and Recommendations for Physical Activity, Medical and Surgical Management. J Assoc Physicians India 2009 Feb;57:163-170

18. Misra A, Singhal N, Sivakumar B, Bhagat N, Jaiswal A, Khurana L. Nutrition transition in India: secular trends in dietary intake and their relationship to diet-related non-communicable diseases. J Diabetes 2011 Dec;3(4): 278-292. 